\title{
The effect of simplifications of a numerical mesh on the results of electromagnetic analysis of the Nuclotron-type cable
}

\author{
Łukasz Tomków ${ }^{1 *}$ \\ ${ }^{1}$ Przedsiębiorstwo Badawczo-Wdrożeniowe Frako-Term sp. z o.o., Toruñ, ul. Morwowa
}

\begin{abstract}
The model of a single Nuclotron-type cable is presented. The goal of this model is to assess the behaviour of the cable under different loads. Two meshes with different simplifications are applied. In the first case, the superconductor in the cable is modelled as single region. Second mesh considers individual strands of the cable. The significant differences between the distributions of the electric current density obtained with both models are observed. The magnetic field remains roughly similar.
\end{abstract}

\section{Introduction}

The Nuclotron-type cable consists of 23 superconducting strands wound around a copper tube, which carries liquid helium to cool down the superconductor below its critical temperature. The assembly is surrounded by several layers of mechanical support including $\mathrm{NiCr}$ wire and Kapton. The structure of the cable is shown in figure 1. The cable of this type was first applied in the superconducting particle accelerator Nuclotron in JINR to construct dipole and quadrupole magnets, hence the name.

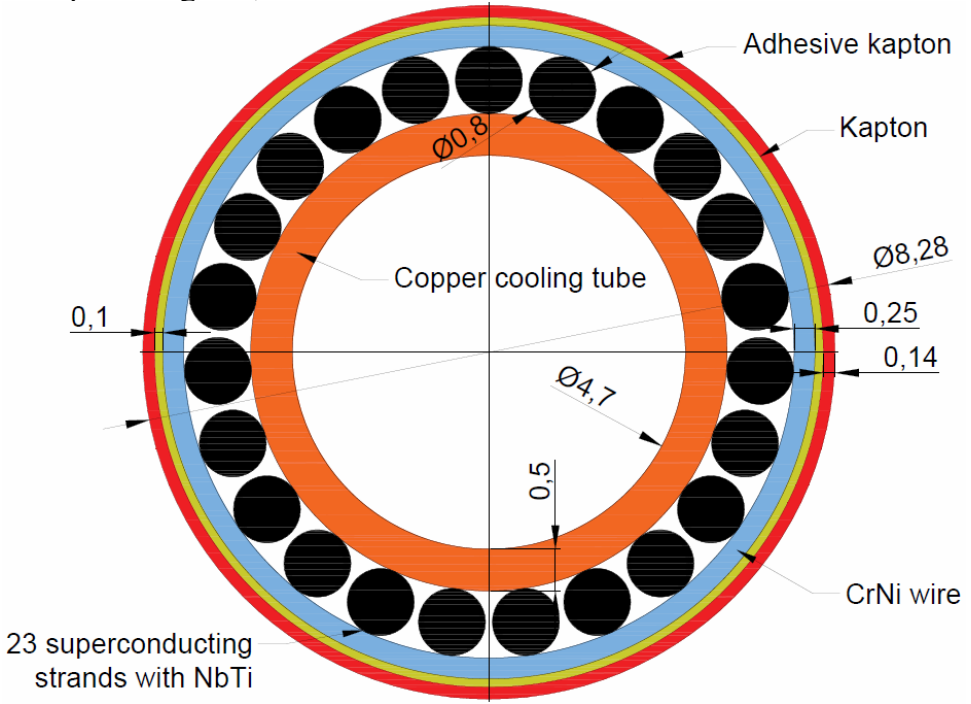

Fig. 1. Structure of a Nuclotron-type cable 
Currently the cables of this type are applied in newly constructed accelerator complexes NICA and FAIR. At FAIR they will be used not only to build magnets magnets, but also at cryogenic transfer lines [1]. The transfer lines will carry electric current and liquid helium between the sections of the accelerator. The superconducting material applied in the cable is NbTi. In this work, the model of the Nuclotron-type cable for the application at the line is presented. The results obtained using two different meshes are considered.

\section{Methods}

The model used in the analysis calculates magnetic field and the electric current distribution in the cable. To find these values the $\mathrm{H}$-formulation is used. The form of the formulation used for the described model was described by Brambilla et al. [2]. The model is two-dimensional with two components of the magnetic field and a single component of electric current density and electric field. To account for high non-linearity the formula for electric field $E$ takes the form (1).

$$
\boldsymbol{E}=\left(\frac{J-J_{c}}{J_{c}} \cdot \mathcal{H}\left(J-J_{c}\right)\right)^{n}
$$

$\boldsymbol{E}$ in this formula is electric field, $J$ is the local density of electric current found as the curl of the magnetic field. $J_{c}$ is critical current density found with Bottura fit [3]. Density of superconducting strands is added as an additional regulating parameter in the calculations of critical current density. In this model the thermal dependence of the critical current density on temperature is not considered. $\mathcal{H}$ is the Heaviside function, which attributes the value of 0 if the value under the function is lower than 0 and 1 if it is higher. $n$ is the exponent of power law.

The second basic formula for the model is Faraday's law giving the interaction between magnetic field and electric field (2).

$$
\frac{\partial B}{\partial t}+\nabla \times \boldsymbol{E}=0
$$

In this formula $\boldsymbol{B}$ is the magnetic induction.

The two meshes used in the analysis are shown in figure 2 . The one to the left leads to more complex and longer analysis with each strand modelled individually. The right mesh models the superconductor as a single region. The calculations performed using the latter one are significantly faster.
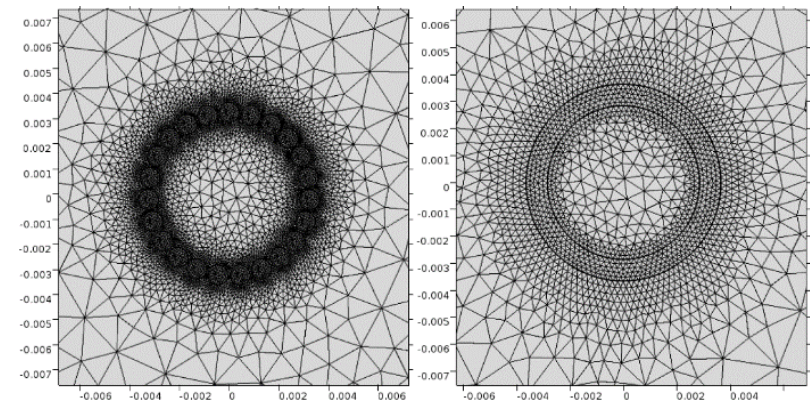

Fig. 2. Meshes applied for the electromagnetic analysis 
The boundary condition for the model is the value of the magnetic field on the external wall of the model. It is calculated based on the magnetic field generated by the point current. In the sufficient distance from the centre of the cable it is a good estimation.

The magnetic field varies with time to reflect the operation of the accelerator in the most demanding testing regime. In this regime the triangular current is applied, which rises from $0 \mathrm{kA}$ to $13 \mathrm{kA}$ with the frequency of $1 \mathrm{~Hz}$. That gives the rate of increase of the current of $26 \mathrm{kA} / \mathrm{s}$.

The additional constraint of the total carried current (expressed as an integral of the current density over the area) is placed on superconducting region. This further ensures the proper value of the carried current.

\section{Results and discussion}

The model yielded the results consistent with initial predictions and literature data. Figure 3 shows the results of the calculations for the magnetic induction with more complex mesh. Figure 4 shows the results on magnetic induction obtained with the simpler mesh.

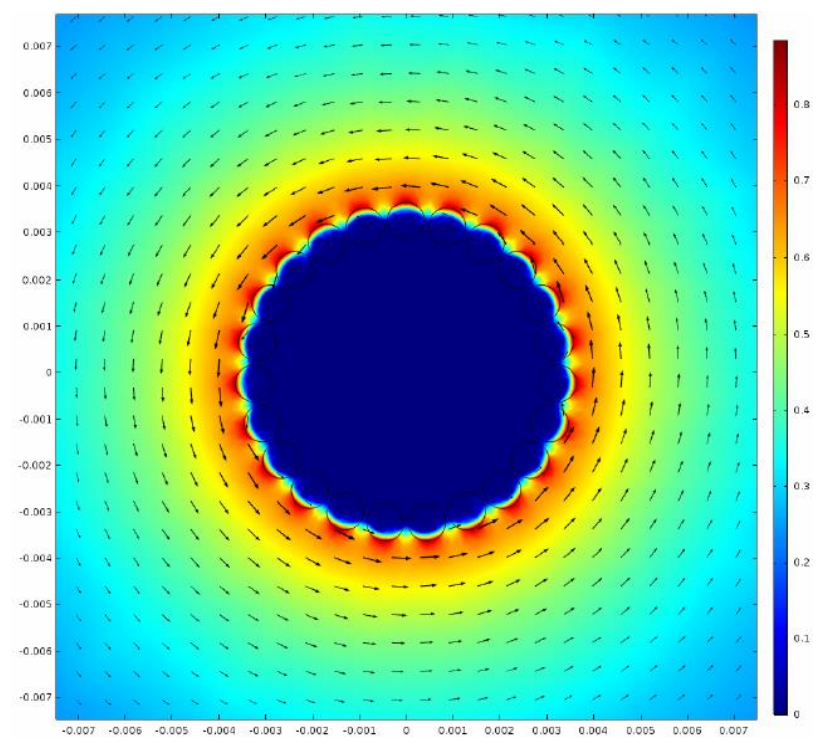

Fig. 3. Magnetic induction in $\mathrm{T}$ calculated with the complex mesh at $t=0.3 \mathrm{~s}$

The magnetic induction can be seen to decrease inside the cable as expected. In the case of the more complex mesh some irregularities in the vicinity of the strands can be observed. Further from the ring of the strands the field becomes regular again, similarly to the case of simpler mesh. The differences disappear less than $0.1 \mathrm{~mm}$ from the strands. Therefore, they do not affect any objects located outside of the cable. It shows that the simplified model is sufficient to calculate the distribution of magnetic field and perform the electromagnetic analysis. The results are very clear and free from the numerical artefacts. 


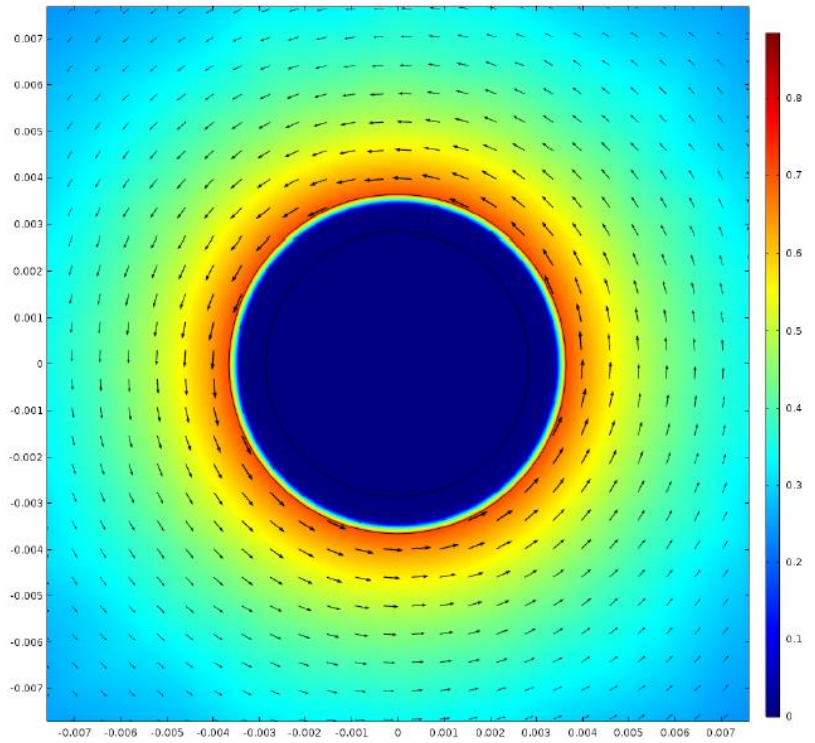

Fig. 4. Magnetic induction in T calculated with the simpler mesh at $t=0.3 \mathrm{~s}$

Figures 5 and 6 present the analogical results of the calculations of electric current density.

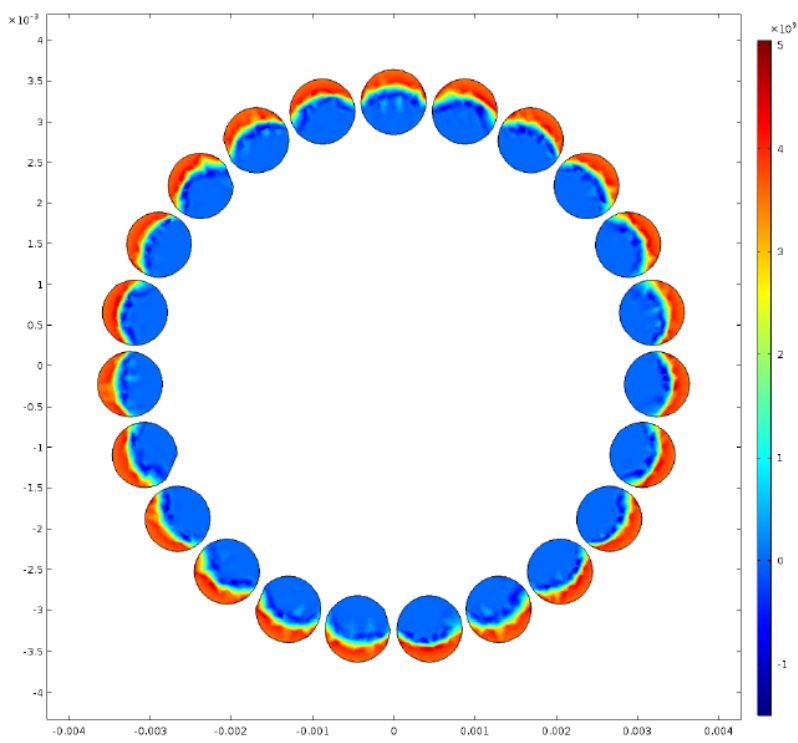

Fig. 5. Electric current density in $\mathrm{A} / \mathrm{m}^{2}$ calculated with the complex mesh at $t=0.3 \mathrm{~s}$ 


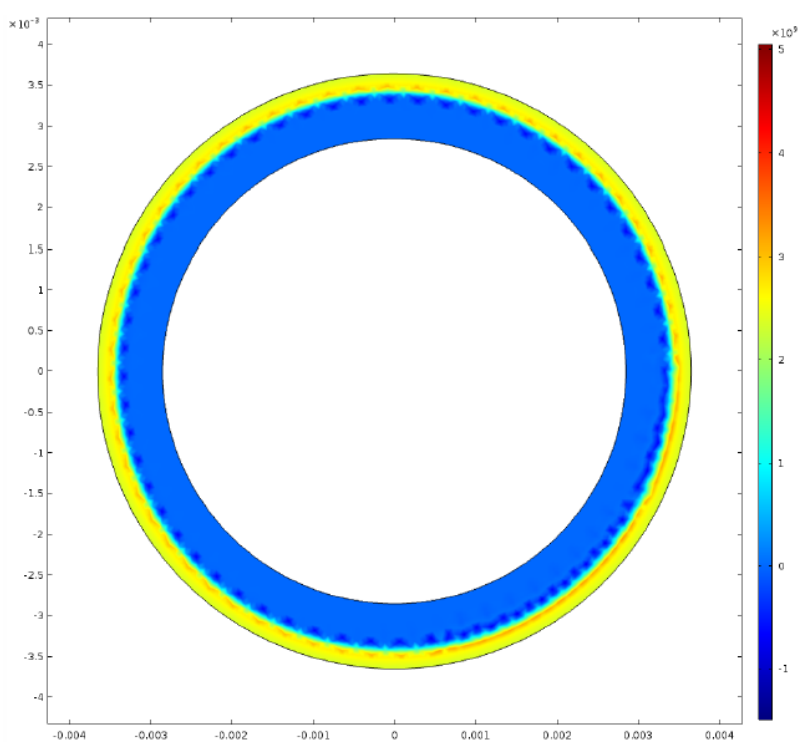

Fig. 6. Electric current density in $\mathrm{A} / \mathrm{m}^{2}$ calculated with the simpler mesh at $t=0.3 \mathrm{~s}$

Significant differences between the results from two analyses can be observed. In the case with more complex mesh the maximum value of the current is significantly higher. It can be expected, as the transport area in the second model is significantly larger. However, that means that the calculated heat generation will be significantly different. Therefore the simpler mesh cannot be applied for thermal analysis of the cable or the calculation of losses occurring during the operation.

Some numerical artefacts can be observed on the interface between the current-carrying region and the region with zero magnetic field. This is especially pronounced in the model obtained with more complex mesh. Their area is relatively small, therefore they do not affect strongly the integral of the current density.

\section{Conclusions}

The simpler mesh cannot be applied for the thermal analysis of the cable due to the significant differences occurring in this model. However, it is well fit for the electromagnetic analysis. The irregularities disappear well within the volume of the cable. The calculations of interactions between the cables in the line will be valid. It is especially important in the assessment of the quality of power delivered to the magnets. It strongly affects the particle beam as well as the safety of operation of the accelerator.

\section{References}

1. A. Bleile, E. Fischer, W. Freisleben, A. Mierau, P. Schnizer, and P. Szwangruber Physics Procedia 67 (2015)

2. R. Brambilla, F. Grilli, and L. Martini Supercond.Sci. Technol 20 (2007)

3. L. Bottura IEEE Transactions on Applied Superconductivity 10 (2000) 